\title{
Position Control of Ultrasonic Motor using Ant Colony Optimization Type Neural Network
}

\author{
Shenglin $\mathrm{Mu}^{\mathrm{a}}$, Satoshi Shibata ${ }^{\mathrm{a}}$, Yamamoto Tomonori ${ }^{\mathrm{a}}$, Kanya Tanaka ${ }^{\mathrm{b}}$, Shota Nakashima ${ }^{\mathrm{c}}$ \\ ${ }^{\text {a }}$ Graduate School of Science and Engineering, Ehime University, 3 Bunkyou-chou, Matsuyama 790-8577 Japan \\ ${ }^{b}$ Department of Electronics and Bioinformatics, School of Science and Technology, \\ Meiji University, Kawasaki, Japan \\ ${ }^{c}$ Graduate School of Sciences and Technology for Innovation, Yamaguchi University, Ube, Japan \\ *Corresponding Author: mu.shenglin.du@ehime-u.ac.jp
}

\begin{abstract}
Along with the progress of computation capability in computers, Artificial Intelligence (AI) are developing in a great pace in recent years. They are widely developed to various industrial fields, especially to the control engineering fields. Among them, there are some bio-inspired algorithms with very attractive characteristics, such as Neural Network (NN), Genetic Algorithms (GA), and Ant Colony Optimization (ACO), etc. In this research, as an intelligent scheme in control application, the intelligent algorithms of neural network algorithm combined with ACO was introduced to a position control scheme for Ultrasonic Motor (USM). Based on traditional PID control, the proposed intelligent scheme offers automatic gain tuning ability in position control for compensating the characteristic changes and nonlinearity of USM. The effectiveness of the proposed method was verified according to simulation study.
\end{abstract}

Keywords: Ultrasonic Motor, PID Control, Neural Network, Ant Colony Optimization.

\section{Introduction}

In recent years, Artificial Intelligence (AI) is an important field with perspective in various applications. Especially to the industrial applications, a lot of approaches have been proposed and introduced. Among the approaches, artificial Neural Network (NN) plays an important role. It is widely used in mechanics, electronics, and informatics. Its application in control engineering constructs a significant part as a representative example in intelligent control. Typically, the training method of Back Propagation (BP) is the considered as the basic learning in NN. It has been confirmed effective in various applications. However, owing to the requirement of plant's derivative information in the learning process and sensitivity to initial weight Therefore, some other learning algorithms have been developing for $\mathrm{NN}$ (1).

Meanwhile, other intelligent algorithms such as evolutionary algorithms (Genetic Algorithm: GA, Evolutionary Strategy: ES, etc.), and swarm intelligence algorithms (Particle Swarm Optimization: PSO, Ant Colony Optimization: ACO, etc.) are developing rapidly with attractive features in computation. They are considered as good alternative of traditional BP learning method in $\mathrm{NN}^{(2}$, 3). In our previous research, PSO was introduced in NN type PID to obtain optimal control gains for Ultrasonic Motor (USM) control. The proposed algorithm shows excellent features in position control without derivative information of USM. In this research, to investigate the properties of swarm intelligence algorithms in NN's learning, a variation of ACO algorithm for real number optimization is introduced in the position control of USM. The characteristics of the proposed method is studied according to simulation study. The paper is organized as follows. In Sect. 2, USM, USM servo system, and the PID control of USM in the servo are introduced. The proposed method with ACO and NN are introduced in Sect. 3. The experimental study is implemented in Sect. 4. In Sect. 5 , the conclusions are given.

\section{USM and Its PID Control}

\section{$2.1 \quad$ USM}

USM is a kind of friction driven electronic motors applied 


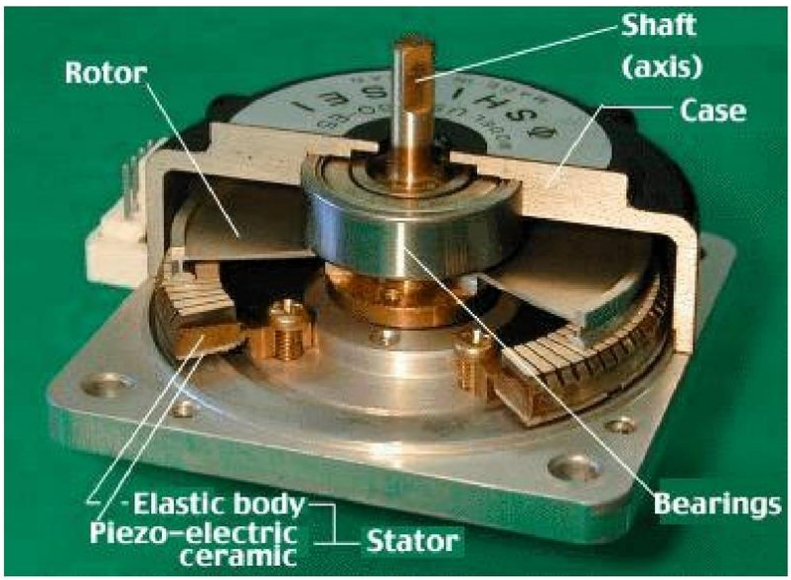

Fig. 1. Cutaway view of travelling wave USM.

in various devices in aerospace engineering, such as satellites, and Mars probers; in optical instruments, such as microscopes, and auto focus lens; in robotics, such as apace manipulators, and aerial robots, etc. ${ }^{(4,5)}$ Figure 1 shows the cutaway view of USM. Different from conventional electronic motors, the core driving components of USMs are the rotor and the stator. When alternate current is added on the piezoelectric ceramic in the stator, elliptical motion of particles in elastic body will be generated. The propagation of the motion leads to the friction generated on the surface between the stator and the rotor. The rotor will be driven into running towards the opposite direction of the friction. The reason why the kind of actuators is so widely used is that there are many excellent characteristics of USMs comparing with conventional electromagnetic motors, such as, quick response, noiseless, compactness, low speed, high torque, no magnetism and so on. However, the control of USM is not easy to achieve owing to the non-linearity and characteristics caused by the friction driven.

\subsection{PID control of USM}

To confirm the effectiveness of proposed method, the USM servo system is constructed and introduced in this section. In the USM servo system for position control, the USM, Laser Rotary Encoder, and the Electro-magnetic brake are connected on a common rotating shaft. Figure. 2 shows the signal flow of USM servo system. First, I/O Board on the Personal Computer send the input signal voltage to the Direct Digital Synthesis (DDS) and the Peripheral Interface Controller (PIC). Second, sine wave will be sent to the Booster Circuit from input signal voltage. Alternating Current $(\mathrm{AC})$ is sent to each electronic component for driving oscillation on the rotor. Laser rotary encoder detects angle of rotation while driving USM. Angle of rotation is

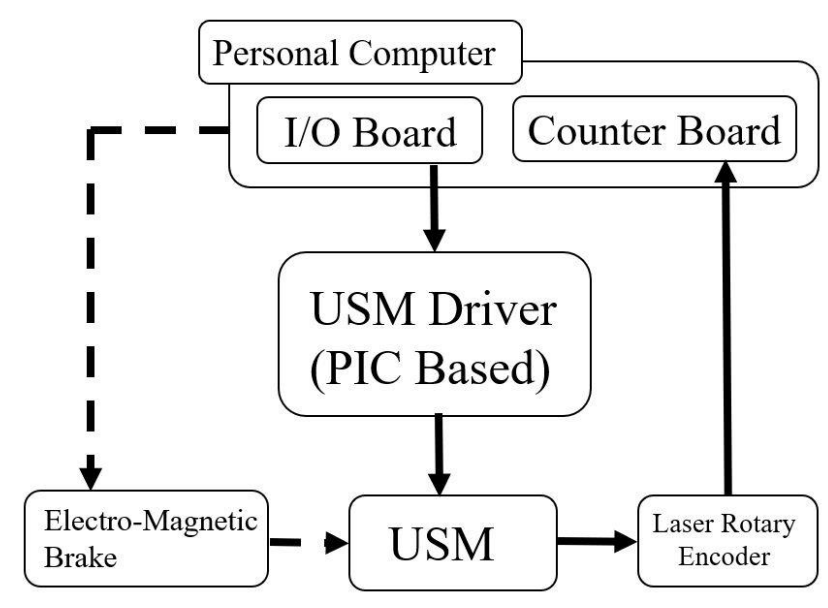

Fig. 2. The signal flow of USM servo system

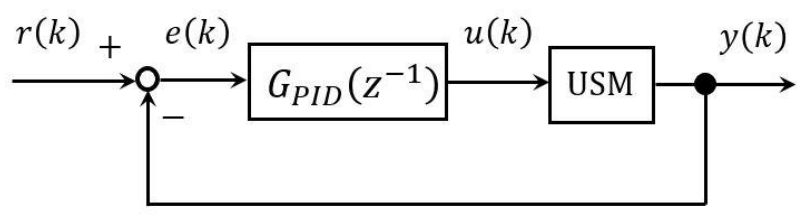

Fig. 3. Block diagram of PID control for USM

sent to the Counter Board as counter values. Personal Computer calculate necessary voltage from the counter values. I/O Board outputs input signal voltage again and repeat the flow until the outputs by the program finish.

PID control is an important control method in various industrial applications ${ }^{(6,7)}$. Figure 1 shows the basic scheme of PID control for USM. In the scheme, $r(k), u(k)$ and $y(k)$ are the object input, the input and the output, respectively. $G_{P I D}\left(z^{-1}\right)$ is the PID controller. The input $u(k)$ is synthesized as

$$
\begin{aligned}
u(k)= & u(k-1)+\left(K_{P}+K_{I}+K_{D}\right) e(k) \\
& -\left(K_{P}+2 K_{I}\right) e(k-1)+K_{D} e(k-2)
\end{aligned}
$$

where $K_{P}, K_{I}$ and $K_{D}$ are the gains of the PID controller. By tuning the PID gains, the control performance can be adjusted. In the scheme, the error denoted by $e(k)$ is expressed as

$$
e(k)=r(k)-y(k)
$$

The PID controller $G_{P I D}\left(z^{-1}\right)$ can be denoted as shown in Eq. (3).

$$
G_{P I D}\left(z^{-1}\right)=\frac{K_{P}\left(1-z^{-1}\right)+K_{I}+K_{D}\left(1-z^{-1}\right)^{2}}{1-z^{-1}}
$$




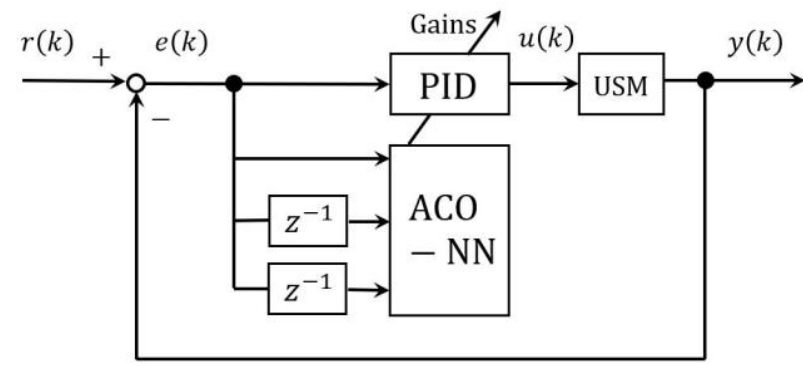

Fig. 4. Control scheme of the proposed method

\section{Proposed method using ACO type NN}

\subsection{Proposed Scheme of NN}

According to the application of $\mathrm{NN}$ in previous research, good control performance could be obtained by making fine tuning of the PID gains in USM control ${ }^{(8)}$. However, since the USM is a non-linear plant without mathematical model, satisfactory performance is difficult to obtain by fixed gain type PID control. Meanwhile, characteristics of USM vary according to running conditions. Fixed gain type PID controller cannot deal with the characteristic changes of USM. To obtain good control performance of USM, gain tuning of PID control requires intelligent tuning according to characteristic changes. Therefore, the proposed intelligent method using ACO and NN is designed in this research as Fig. 4 shows. Based on conventional PID controller for USM, the intelligent model - ACO type $\mathrm{NN}$ is employed for updating the PID gains on-line. As we see in the scheme, the input of the intelligent controller is the discrete time signal of $I(k)=[e(k), e(k-1), e(k-2)]$. The topologic structure of the NN controller is shown in Fig. 5. There are 3 layers in the structure with a single hidden one containing 6

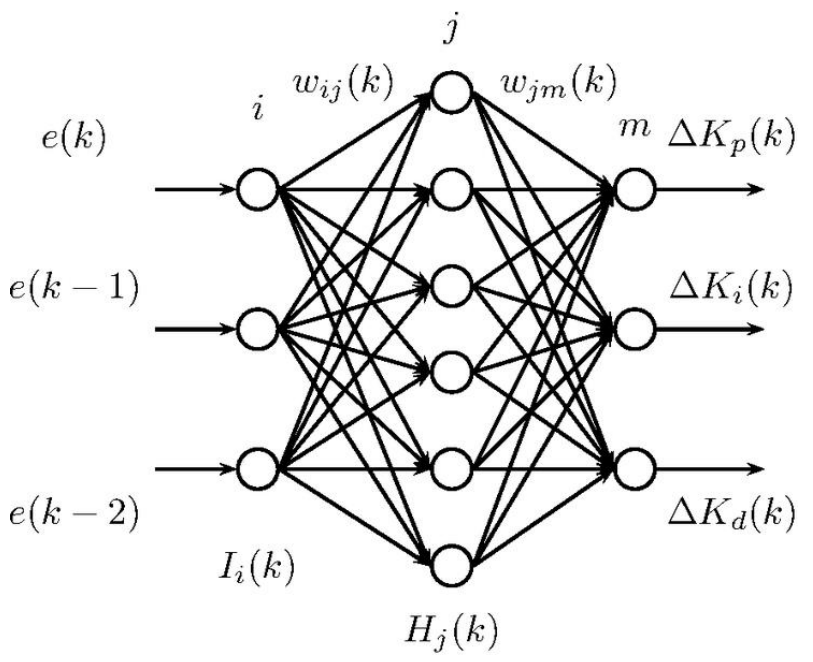

Fig. 5. Structure of NN in proposed method neurons, and 3 neurons in input and output layers, respectively. The output of NN is the variation of gains for PID controller which can be expressed as $\left[\Delta K_{P}, \Delta K_{I}, \Delta K_{D}\right]$. The scheme is designed for tuning PID gains automatically to achieve good performance in USM control. As the activation function, the hyperbolic tangent function shown in Eq. (4) was introduced.

$$
f(x)=\tanh (x)=\frac{e^{x}-e^{-x}}{e^{x}+e^{-x}}
$$

According to the proposed scheme, the weight updating with learning algorithms could lead the intelligent scheme to fine control performance minimize the error $e(k)$ in positioning. To make the weight updated in optimal way, $\mathrm{ACO}_{\mathrm{R}}$, which is a variation of conventional ACO algorithm for real number, was introduced.

\subsection{Ant Colony Optimization and $\mathrm{ACO}_{\mathrm{R}}$}

$\mathrm{ACO}$ is one of the Swarm Intelligence algorithms which was formalized as an effective optimal tool for solving combinational optimization problems by Dorigo and his coworkers ${ }^{(4)}$. The inspiring source of ACO is the foraging behavior of some ant species. These ants deposit pheromone on the ground to mark some favorable path that should be followed by other members of the colony ${ }^{(9-11)}$. However, the application of the conventional ACO in optimization of continuous problems was not easy. In reference (12) the work from Socha and his co-workers improved the application of ACO into real number with the advanced algorithm of $A C O R$.

In the algorithm of $\mathrm{ACO}_{\mathrm{R}}$, the solutions contained by ants are obtained using Probability Density Function (PDF). The popular function applied as a PDF is the Gaussian function. The defined Gaussian kernel, which is weighted combined with multiple one-dimensional Gaussian function $g_{l}^{i}(x)$ can be expressed as

$$
G^{p}(x)=\sum_{l=1}^{q} \omega_{l} g_{l}^{p}(x)=\sum_{l=1}^{q} \omega_{l} \frac{1}{\sigma_{l}^{i} \sqrt{2 \pi}} e^{-\frac{\left(x-\mu_{l}^{p}\right)^{2}}{2 \sigma_{l}^{p^{2}}}}
$$

where $p=1, \ldots, n$ is the number of dimensions in the problem. $\omega$ are the weights associated with the individual Gaussian functions. $\mu^{p}$ are the means of the Gaussian functions, and $\sigma^{p}$ are the standard deviations.

In this application, we consider the vector of weights in $\mathrm{NN}$ as the dimensions in a single solution of $\mathrm{ACO}_{\mathrm{R}}$. The archive of solutions can be expressed as 


$$
\mu^{p}=\left\{\mu_{1}^{p}, \ldots \mu_{q}^{p}\right\}=\left\{w_{1}^{p}, \ldots w_{q}^{p}\right\}
$$

In this application, the weights in NN controller are designed as the information in the dimensions. The number of dimensions for the NN weight is $q=36$ for the full connections in the topological structure. As introduced in reference (12), the value of the standard deviation $\sigma_{l}^{i}$ was established by the following equation

$$
\sigma_{l}^{p}=\xi \sum_{e=1}^{q} \frac{\left|w_{e}^{p}-w_{l}^{p}\right|}{q-1}
$$

$\xi$ in the $\mathrm{ACO}_{\mathrm{R}}$ algorithm influences the way the long-term memory is used.

\section{Simulation Study}

To introduce the proposed intelligent scheme in real-time servo system of USM. The simulation study on existent model was implemented. Equation (1) shows the expression of the plant.

$$
\begin{aligned}
y(k)= & 0.0266 u(k-1)+0.560 y(k-1) \\
& +0.439 y(k-2)+0.01
\end{aligned}
$$

A step signal with a constant amplitude of 45 degree was used as objective input $r(k)$. The control input was set the same as the existent USM servo system with the range of [$0.5,0.5]$. The weights were initialized using random real numbers according normal distribution within the range of [1.0, 1.0]. The information was contained by the ants in 36 dimensions as defined in the proposed method. The ant number was set to 10 for convenience. The solutions contained by the ants will be bring into the $\mathrm{NN}$ scheme for computing the variation of PID gains. To evaluate the performance of the possible solutions contained by the ants, the fitness of the solutions was designed as

$$
\text { fitness }=\frac{1}{1+e(k)^{2}}
$$

The ants with high fitness approaching to 1.0 will be ranked higher in the ant archive. By employing the proposed method, the response obtained in the simulation can be seen in Fig. 5. It was observed that the output of the model approached to the objective signal rapidly without overshoot. However, there were some oscillations at the steady-stated stage in the simulation. The variation of the gains in the proposed scheme is shown in Fig. 6. The gains varied in a short period and converged to certain range. The weights contained by ants in the $\mathrm{ACO}_{\mathrm{R}}$ algorithm were evaluated by Eq. (9). A

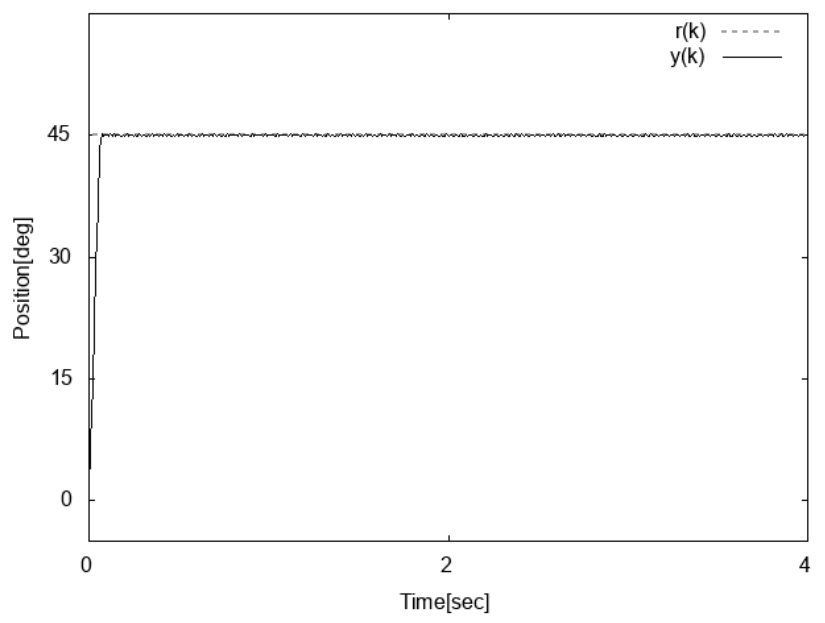

Fig. 5 Response of the proposed intelligent scheme.

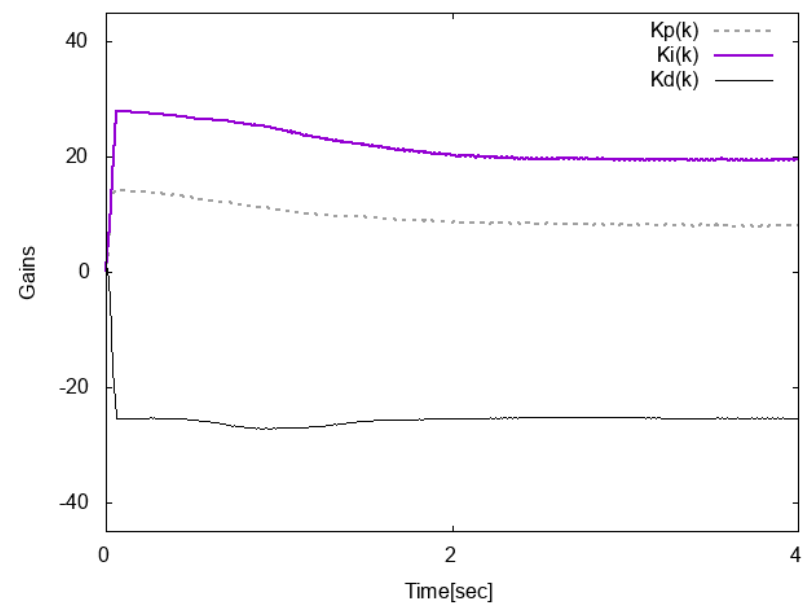

Fig. 6 Gain variation of the proposed intelligent scheme.

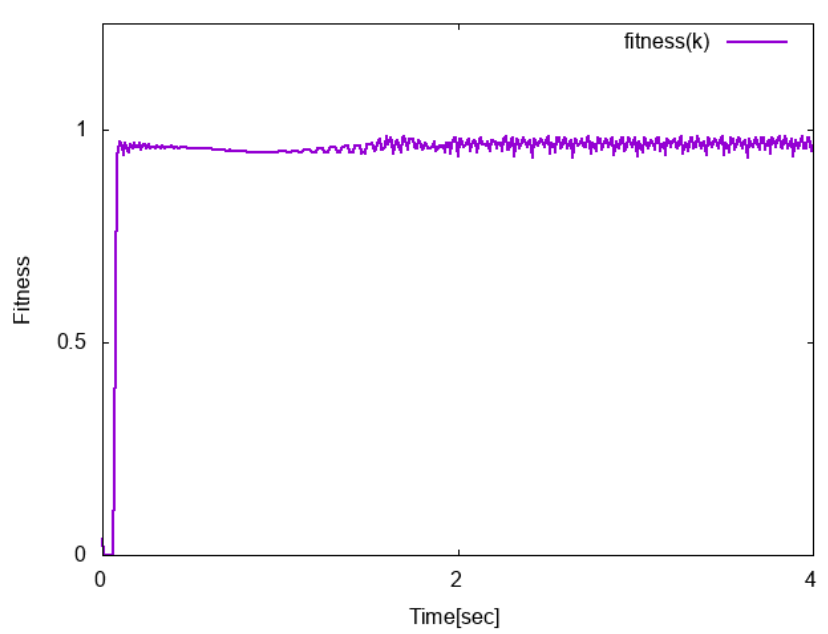

Fig. 7 Gain variation of the proposed intelligent scheme. 
reprehensive fitness of an ant is shown in Fig. 7. The oscillation at the steady-state stage we can see in the figure, validated our observation in Fig. 5. At this stage, the optimal adjustment in the algorithm is still expected for compensating the oscillation.

\section{Conclusions}

In this research, an ACO algorithm is introduced to the $\mathrm{NN}$ learning in the position control of USM based on PID control. The effectiveness of the proposed method was confirmed according to simulation results. In the following stage the application in real time environment on existent USM servo system will be implemented. Meanwhile, optimal parameters in application will be investigated.

\section{References}

(1) J. F. Kolen and J. B. Pollac: "Back Propagation is Sensitive to Initial Conditions", NIPS-3 Proceedings of the 1990 conference on Advances in neural information processing systems 3, pp 860-867, 1990

(2) C. Juang, "A Hybrid of Genetic Algorithm and Particle Swarm Optimization for Recurrent Network Design", IEEE Transactions on Systems, Man, and Cybernetics Part b: Cybernetics, Vol. 34, No. 2.

(3) K. Shimizu, J. Komiyama, M. Toyota, "Weight Optimization for Neural Network Combined with Genetic Algorithm”, DEIM Forum, P4-6, 2018

(4) C. Zhao, Ultrasonic Motor - Technologies and Applications, Science Press Beijing and Springer Verlag Berlin Heidelberg, Beijing, 2011.
(5) S. Mu and K. Tanaka, Intelligent IMC-PID control using PSO for ultrasonic motor, International Journal of Engineering Innovation and Management, vol.1, pp.6976, 2011.

(6) K. Astrom, T. Hagglund, C. Hang and W. Ho, Automatic tuning and adaptation for PID controllers - A survey, Control Engineering Practice, vol.1, pp.699-714, 1993.

(7) K. J. Astrom and T. Hagglund, Revisiting the ZieglerNichols step response method for PID control, Journal of Process Control, vol.14, pp.635-650, 2004.

(8) K. Tanaka, Y. Yoshimura, Y. Wakasa, T. Akashi, M. Oka and $\mathrm{S}$. Mu, Variable gain type intelligent PID control for ultrasonic motor, The Japan Society Applied Electromagnetics and Mechanics, vol.17, pp.107-113, 2009 (in Japanese).

(9) M. Dorigo, M. Birattari, and T. stiitzle, Ant Colony Optimization, Artificial Ants as a Computational Intelligence Technique, Universite Libre de Bruxelles, , IEEE Computational Intelligence Magazine, 2006.

(10) M. Dorigo, G. Di Caro, and L.M. Gambardella, “Ant algorithms for discrete optimization," Artificial Life, vol. 5, no. 2, pp. 137-172, 1999.

(11) M. Mavrovouniotis and S. Yan, Training neural networks with ant colony optimization algorithms for pattern classification, Soft Computing, Vol. 19, Issue 6, pp1511-1522, June 2015.

(12) K. Socha and C. Blum, An ant colony optimization algorithm for continuous optimization: application to feed-forward neural network training, Neural Computing \& Application (2007) 16:235-247 DOI 10.1007/s00521-007-0084. 